\title{
Impact of COVID-19 on rehabilitation experiences of physiotherapists
}

\author{
Pınar Kaya Ciddi* and Gülay Aras Bayram \\ Physiotherapy and Rehabilitation Department, Faculty of Health Sciences, Istanbul Medipol University, Istanbul, \\ Turkey
}

Received 27 April 2021

Accepted 25 August 2021

\begin{abstract}
.
BACKGROUND: As the COVID-19 pandemic progressed, pressures on almost all health sectors in many countries increased, and physiotherapy interventions were canceled for various reasons.

OBJECTIVES: The aim of this study was to determine the status of physiotherapists who interrupted their services due to the pandemic and investigate procedures adopted by physiotherapists during face-to-face practice.

METHOD: The measuring tool was an online survey administered via Google Forms between January 27 and February 27, 2021. In this descriptive cross-sectional study, the sample consisted of 558 physiotherapists, questions about their clinical experience during the pandemic were answered, and descriptive statistics were examined.

RESULTS: Of the physiotherapists, 351 (62.9\%) suspended their services due to pandemic, while 207 (37\%) of all participants worked without suspending their services since the beginning of the process. Among participants, $303(54.3 \%)$ needed education to use telerehabilitation methods, and $315(56.5 \%)$ monitored their patients with remote communication methods. Hand washing (86.6\%), disinfectant (85.3\%), gloves (76.5\%) and masks (86.6\%) were the most common protective measures. CONCLUSIONS: Most physiotherapists had their face-to-face practice interrupted for a short time due to the COVID-19 outbreak, but they continue to treat all disease conditions in spite of inherent physical intimacy and increased risk of infection.
\end{abstract}

Keywords: Coronavirus, COVID-19, health professionals, preventive measures, telehealth

\section{Introduction}

The COVID-19 outbreak had a major impact on the acute sector of healthcare services (especially emergency services, intensive care units, laboratory and imaging services) from the very beginning, and the burden on these services is still increasing [1, 2]. As the pandemic progressed, pressure on almost all healthcare sectors increased [3-5], including the areas of post-acute care and rehabilitation.

\footnotetext{
*Address for correspondence: Pınar Kaya Ciddi, Assistant Professor, Physiotherapy and Rehabilitation Department, Faculty of Health Sciences, Kavacík South Campus, Istanbul Medipol University, Atatürk Caddesi no. 40/16, 34815, Kavacík/ Beykoz/İstanbul, Turkey. E-mail: pkaya@medipol.edu.tr.
}

Physiotherapy and rehabilitation are a subacute specialty and the main effect of restrictions on this specialty was the cancellation of rehabilitation interventions for various reasons, and it was reported the long-term continuity of the restrictions will have negative consequences for function and disability [5]. A study revealed that up to 2.2 million people in Europe interrupted their rehabilitation treatments due to the pandemic [6]. Many rehabilitation specialists have reasonable concerns about whether rehabilitation services can be adequately reorganized [5] when this emergency is over and that physiotherapists will have to deal with very large groups of patients in need of rehabilitation [7].

During physiotherapy and rehabilitation interventions, specific and inherent aspects such as the need 
for long-term and close interaction between patients and professionals, frequent difficulties in communicating with the patient (due to cognitive difficulties, consciousness disorders, pediatric patients, etc.), and the need to involve family members or other persons in the provision of rehabilitation services can lead to difficulties in aligning therapeutic needs with the necessary measures to protect professionals from infection and prevent the spread of infection [1]. Because asymptomatic individuals were shown to spread the infection, physiotherapists exposed to the virus may pose a risk to older patients and other patients with comorbidities [8].

To properly address these aspects and reduce risks, the use of disposable personal protective equipment which should be changed between patients [1, 9-11], reductions in the number of patients and therapists in the same physical space, adjustment of therapy spaces according to distance needs [8] and the use of technology-based digital physiotherapist applications to communicate and guide patients are recommended by many international organizations [12]. However, it is important to strengthen the academic and educational content knowledge of rehabilitation professionals about assessment and treatment with telerehabilitation for these implementations [4].

With the impact of such factors and the rapid progression of the pandemic, the pressure and impact on rehabilitation services are constantly and rapidly changing over time and in different local conditions $[8,13]$. However, these changes are not tailored to the specific needs of rehabilitation activities [7]. With the study, the assumption was that the needs which will arise after the pandemic can be predicted by analyzing the effect of the pandemic and clinical reorganization on physiotherapy and rehabilitation clinics where physiotherapists work during the COVID-19 pandemic. The purpose of this study is to determine the situation of physiotherapists whose services were interrupted due to the COVID-19 outbreak and to investigate the procedures performed by currently working physiotherapists in Turkey.

\section{Materials and methods}

This was a descriptive cross-sectional and single blind study to evaluate the clinical behavior of physical therapists working in Turkey during the pandemic as the target population. Medical ethical approval was obtained from the clinical research ethics committee of Istanbul Medipol University with number E-10840098-772.02-2682. The study was registered on ClinicalTrials.gov under no. NCT04836039.

After all participants were informed about the aims of the study and they were ensured that their answers would be kept anonymous, consent was obtained from all participants. Inclusion criteria for the research were having a graduate degree in physiotherapy and rehabilitation in Turkey, at least 6 months clinical experience in this field and agreeing to participate in this research. The population consisted of physiotherapists of all ages, regardless of gender.

\subsection{Measurement instrument}

Data collection took place between January 27 and February 27, 2021. An online survey was administered via Google Forms as a measurement tool. The questions and statements in the questionnaire were developed by the authors based on previous studies $[7,14]$ and were critiqued (by physiotherapy professors) based on an objective approach for the analysis of statements used for measurement. Afterwards, the questionnaire was sent to three physiotherapists and a preliminary evaluation was completed. Afterwards, the questionnaire was distributed to physiotherapists via WhatsApp, e-mail and social networks. This questionnaire included questions about gender, age, duration of professional experience, region of professional practice, physiotherapy subgroup usually practiced (more than one could be selected), employment status, continuing face-to-face practice, and protection methods adopted.

With the questionnaire, physiotherapists were asked whether they were involved in rehabilitation services for patients with COVID-19, and if so, what clinical situations required physiotherapy and rehabilitation for patients with COVID-19. While performing these practices, physiotherapists were asked whether they developed or benefited from exercise, protocols and/or guidelines in the form of audiovisual material for rehabilitation management and treatment of patients with COVID-19.

Questions were asked about whether physiotherapists who interrupted their face-to-face clinical practices for various reasons started to monitor their patients remotely; if so, which communication method they used, whether the number of weekly sessions were similar to normal and the charge for these sessions. Finally, the questionnaire concluded with questions about whether they considered themselves 
competent in terms of implementing telerehabilitation, had received education on this subject to improve themselves, whether they needed education, and if so, what subjects they required information about within the scope of telerehabilitation.

\subsection{Data analysis}

Statistical analysis was performed with IBM SPSS statistics, version 22 and descriptive statistics were examined. Chi-square was used to test for significant associations between categorical variables. Statistical significance was set at 0.05 .

\section{Results}

Forty-nine of the 607 questionnaires sent to participants were excluded because there were errors in completing them. The sample consisted of 558 physiotherapists and the demographic characteristics of them are presented in Table 1.

For generally applied physiotherapy subgroups, physiotherapists were offered the option to choose more than one area. Most of the physiotherapists worked in the field of pediatric rehabilitation $(n=342,61.3 \%)$, and then the following areas, adult neurological rehabilitation $(n=300,53.8 \%)$, musculoskeletal system $(n=297,53.2 \%)$, geriatric rehabilitation $(n=165,29.6 \%)$, pulmonary physiotherapy $(n=81,14.5 \%)$, cardiovascular rehabilitation $(n=48,8.6 \%)$, palliative care $(n=27,4.8 \%)$, urogynecology $(n=24,4.3 \%)$ and other intervention areas $(n=8,1.4 \%)$. Among physiotherapists, 358 (64.1\%) worked in hospitals and clinics, 136 (24.4\%) worked in centers for disabled individuals, 49 (8.8\%) were self-employed (working in their own center) and 15 $(2.7 \%)$ were currently unemployed (Table 1$)$.

In terms of patient groups whose continuity of treatment decreased in the last year, the main groups identified were pediatric patients $(n=324$, $58.1 \%)$, adult neurological patients $(n=288,51.6 \%)$ and patients with musculoskeletal injuries $(n=192$, $34.4 \%$ ) (Table 1). Among physiotherapists, $62.9 \%$ $(n=351)$ reported that their services stopped for $3.23 \pm 0.12$ months due to the pandemic in and after April 2020. It was reported that $76.9 \%(n=270)$ of physiotherapists who stopped their services for a while during the pandemic are currently working face to face (Table 1). It was observed that $37 \%$ $(n=207)$ of all participants worked without interruption since the beginning of the pandemic. Among physiotherapists, $18.8 \%(n=66)$ of those who interrupted their clinical services stated that their clinics were still closed at the time of the study, but that they would continue when their clinics reopened. Six (1.7\%) physiotherapists who interrupted their clinical services reported that their clinics stopped providing service completely and they were unemployed, while $9(2.6 \%)$ reported that their clinics were currently in service but they were fired (Table 1).

In terms of patients with COVID-19, 27.4\% $(n=153)$ of the physiotherapists performed physiotherapy and rehabilitation for patients with COVID-19 during the pandemic, and Table 1 shows the clinical findings requiring rehabilitation among these patients. The development and utilization of materials for rehabilitation management and treatment of patients with COVID-19 during the pandemic are shown in Table 1. Protective measures adopted by physiotherapists during face-to-face practice are shown in Table 2.

For remote work, $56.5 \%(n=315)$ of the participants used remote communication methods, and the methods used and their rates are shown in Table 3. It was found that $69.5 \%(n=219)$ of the individuals using these methods had the same number of weekly sessions as during face-to-face practice, and $30.5 \%$ $(n=96)$ did it with less frequency. For individuals using these methods, $9.5 \%(n=30)$ of individuals received a fee for their appointments; $60 \%(n=18)$ received the same amount as face-to-face fees and $40 \%(n=12)$ received amounts lower than face-toface fees (Table 3 ).

When the participants were asked whether they had sufficient knowledge to use telerehabilitation methods, they gave the following answers; $38.7 \%(n=216)$ said "no", 32.3\% $(n=180)$ said "enough for me" and $29 \%(n=162)$ said "I have knowledge". It was reported that $48(8.6 \%)$ of the participants attended webinars about telerehabilitation practices in order to use telerehabilitation methods during the pandemic. When asked whether they needed education about telerehabilitation methods, 54.3\% $(n=303)$ needed education. When asked about the topics they were curious about within telerehabilitation education, participants were looking for answers to the following questions; "What are the methods and platforms where I can reach my patients remotely in the most effective way?" $48.8 \%(n=148)$, "What interventions can I apply to my patients remotely via videoconferencing?" 40.2\% ( $n=122)$, and "What are the methods by which I can remotely evaluate my patients?" 27.4\% $(n=83)$ (Table 3$)$. 
Table 1

Participants and work practice characteristics

\begin{tabular}{lcc}
\hline & Mean (SD) & Min-Max \\
\hline Age (years) & $28.11 \pm 0.26$ & $22-58$ \\
Professional experience (years) & $5.14 \pm 6.03$ & $0.5-34$ \\
Variable/Category & $n$ & $\%$ \\
Gender & & \\
$\quad$ - Female & 438 & 78.5 \\
- Male & 120 & 21.5 \\
Region of professional practice $(n=558)$ & & \\
- Aegean region & 38 & 6.8 \\
- Black Sea region & 52 & 9.3 \\
- Central Anatolia region & 58 & 10.4 \\
- Mediterranean region & 34 & 6 \\
- Southeastern Anatolia region & 24 & 4.3 \\
- Eastern Anatolia region & 12 & 2.2 \\
- Marmara region & 340 & 60.9
\end{tabular}

Physiotherapy subgroup usually practiced $(n=558)$

(more than one option can be selected)

- Pediatric rehabilitation

- Adult neurological rehabilitation

- Musculoskeletal diseases

- Geriatric rehabilitation

- Pulmonary physiotherapy

- Cardiovascular rehabilitation

- Palliative care

- Urogynecology

- Other areas of intervention

urrent employment status $(n=558)$

- Hospitals and clinics

- Centers for disabled individuals

- Self-employed (working in their own center)

- Unemployed

What are the patient groups whose continuity

of treatment decreased in the clinic where you work during the pandemic? $(n=558)$

(more than 1 option can be selected)

- Pediatric patients

- Adult neurological patients

- Patients with musculoskeletal injuries

Has your clinical service suspended during the pandemic? $(n=558)$

If your answer is yes: for how long and under what conditions was your clinical service suspended? $(n=351)$

- My clinic stopped service for a while, now I continue to work face-to-face.

- My clinic suspended its service for a while, when it reopens, I will continue.

- My clinic stopped service completely and I was unemployed.

- My clinic is currently in service, I was fired by the workplace.

for COVID patients during the pandemic? $(n=558)$

What were the clinical situations that required physiotherapy intervention for your COVID-19 patients? $(n=153)$ (more than 1 option can be selected)

- Respiratory weakness

- General muscle weakness

- Atrophy

- Fatigue

- Neuropathy 
Table 1

Continued)

\begin{tabular}{lcc}
\hline & Mean (SD) & Min-Max \\
\hline $\begin{array}{l}\text { Have you participated in development team for audio-visual } \\
\text { materials such as exercises, protocols and/or guidelines }\end{array}$ & 60 & 10.8 \\
for rehabilitation management and treatment of & & \\
COVID-19 patients? $(n=558)$ & 100 & 17.9 \\
$\begin{array}{l}\text { Have you used audio-visual materials such as exercises, } \\
\text { protocols and/or guidelines for rehabilitation management } \\
\text { and treatment of COVID-19 patients? }(n=558)\end{array}$ & \\
\hline
\end{tabular}

Table 2

Protective precautions taken during face-to-face practice by physiotherapists

\begin{tabular}{lcc}
\hline $\begin{array}{l}\text { What precautionary methods have } \\
\text { you adopted to protect from the } \\
\text { pandemic in the last 1 year? }\end{array}$ & $n$ & \\
(More than 1 option can be & & \\
selected) $(n=558)$ & & \\
\hline - Hand washing & 483 & 86.6 \\
- Disinfectant & 476 & 85.3 \\
- Surgical mask & 483 & 86.6 \\
- Surgical apron & 57 & 10.2 \\
- Face shield & 196 & 35.1 \\
- Surgical glove & 427 & 76.5 \\
- Social distance & 357 & 64 \\
- Decrease in the number of & 126 & 22.6 \\
$\quad$ patients in the clinic & & \\
- Rotation with colleagues & 84 & 15 \\
- Regular COVID-19 testing & 8 & 1.4 \\
\hline
\end{tabular}

\section{Discussion}

This study includes results about rehabilitation practices during the COVID-19 pandemic regarding physiotherapists in Turkey, such as clinical service interruptions due to restrictions, protective measures used when working face-to-face, patient groups who stopped treatment and remote communication methods used to reach patients. Negrini et al. [6] reported that during the restrictions, outpatient rehabilitation services for chronic diseases were suspended to give priority to intensive and acute care for patients with COVID-19. According to our conclusions, the majority of the clinics where physiotherapists worked during the pandemic stopped service for a while, but they are currently working face-to-face and most physiotherapists continued their service without interruption The sudden loss of income experienced by rehabilitation centers during the pandemic restrictions created a risk for viability of these institutions [8]. For this reason, physiotherapists continued to perform interventions for all disease conditions, while facing an increased risk of COVID-19 infection with physical close contact, which is inherent in their usual practice due to the large number of patients being treated every day [8].

During this process, the timely provision of protective equipment and the intensive use of this equipment by physiotherapists reduced the transmission of infection and helped overcome fear and anxiety $[5,8]$. Secondly, it was reported that reducing the number of patients and therapists in clinics can facilitate social distancing [8]. According to our study, the majority of physiotherapists used some kind of protective measures such as hand washing, disinfectant, use of gloves and masks, but there was less use of gowns and visors, and most importantly, they applied measures such as rotating with their colleagues and reducing the number of patients to a lesser extent. The current situation shows that although the rate of physiotherapists working in rehabilitation services who continue working face-to-face is high in our country, the precaution to reduce the number of patients and therapists reported by previous authors was not adequately followed. It was reported that if the COVID-19 crisis continues for a long time, physiotherapists may start to prefer more therapeutic exercises and avoid manual techniques that require close contact [8]. This situation is far from ideal due to the possibility that many benefits of holistic approaches may be lost, and it is an inevitable fact that physiotherapy and rehabilitation interventions are a basic service for public health [8]. All existing measures should be taken to reduce the possibility of infection for both the physiotherapist and the patient. If the number of patients and therapists cannot be reduced for various reasons, individual preventive measures should be increased and each patient should be evaluated individually in terms of the risks and benefits of the planned intervention.

Alternative methods were adopted to deliver care (remote consultation, telerehabilitation, etc.) while managing the precautions to be taken in order to reduce the risk of infection. It is important to consider using these alternative care options in order to maintain the therapeutic relationship while interrupting 
Table 3

Physiotherapist experiences of communicating with their patients remotely

\begin{tabular}{|c|c|c|}
\hline Variable/Category & $n$ & $\%$ \\
\hline $\begin{array}{l}\text { Have you used remote communication methods with your patients } \\
\text { during the pandemic? }(n=558)\end{array}$ & 315 & 56.5 \\
\hline \multicolumn{3}{|l|}{$\begin{array}{l}\text { If your answer is yes: which method did you use? }(n=315) \\
\text { (more than } 1 \text { option can be selected) }\end{array}$} \\
\hline - video conference & 117 & 37.1 \\
\hline - video sharing (exercise videos, etc.) & 147 & 46.7 \\
\hline - written text message & 123 & 39 \\
\hline - telephone call & 237 & 75.2 \\
\hline \multicolumn{3}{|l|}{$\begin{array}{l}\text { Was your frequency of remote interviews with your patients in the same } \\
\text { number of weekly sessions as in face-to-face meetings? }(n=315)\end{array}$} \\
\hline - Same number of weekly sessions as in face-to-face meetings & 219 & 69.5 \\
\hline - Fewer sessions than face-to-face meetings & 96 & 30.5 \\
\hline $\begin{array}{l}\text { Have you charged a fee for remote interview sessions } \\
\quad \text { with your patients? }(n=315)\end{array}$ & 30 & 9.5 \\
\hline \multicolumn{3}{|l|}{$\begin{array}{l}\text { If your answer is yes: was the fee you charged the same amount } \\
\text { as face-to-face fees? }(n=30)\end{array}$} \\
\hline - Same amount as face-to-face fees & 18 & 60 \\
\hline - Lower fees than the face-to-face amount & 12 & 40 \\
\hline \multicolumn{3}{|l|}{ Do you have enough information to use telerehabilitation methods? $(n=558)$} \\
\hline - "No" & 162 & 29 \\
\hline - "I have some knowledge" & 216 & 38.7 \\
\hline - "Enough for me" & 180 & 32.3 \\
\hline $\begin{array}{l}\text { Have you participated in education (webinar, etc.) to learn how to use } \\
\text { telerehabilitation methods during the pandemic? }(n=558)\end{array}$ & 48 & 8.6 \\
\hline $\begin{array}{l}\text { Do you need for education to learn to use telerehabilitation } \\
\text { methods effectively? }(n=558)\end{array}$ & 303 & 54.3 \\
\hline \multicolumn{3}{|l|}{$\begin{array}{l}\text { What are the topics that you request to be included in the telerehabilitation } \\
\text { education and are curious about? }(n=303) \text { (more than } 1 \text { option can be selected) }\end{array}$} \\
\hline $\begin{array}{l}\text { - "What are the methods and platforms that I can use to reach } \\
\text { my patients remotely in the most effective way?" }\end{array}$ & 148 & 48.8 \\
\hline - "What interventions can I apply to my patients remotely via videoconferencing?" & 122 & 402 \\
\hline - "What are the methods by which I can remotely evaluate my patients?" & 83 & 27.4 \\
\hline
\end{tabular}

the treatment of patients with chronic conditions that are at risk of rapid deterioration in their functional levels if left untreated (such as neurodegenerative diseases, severe conditions in childhood, progressive disease, etc.) [1]. Professionals report that they try to provide telerehabilitation in critical situations or, at the very least, by telephone or video call [15]. Our findings show that patients who had to stop treatment and rehabilitation processes due to the new situation in our country were monitored telemetrically by their physiotherapists. As in other countries [1], despite all these conditions, physiotherapists try to do their best to provide adequate care for their patients, especially those in critical condition due to health conditions and functional limitations. Consistent with the results reported in previous studies [5], many participants in our study mostly used telephone calls as a remote communication method. The participants used video sharing, written text messages and very few participants used video conferencing as other methods. However, many interviews require face-to-face examination. In telephone conversations, the importance of non-verbal communication or body language is lost, and it is clear that clinics should have access to video conferencing methods to overcome this situation [5]. However, these methods involve a difficult learning process that is not suited to the urgent need context and many older patients may find it difficult to use new technologies; involving families and caregivers in treatment sessions can reduce this barrier and increase participation [8]. These strategies will require legal regulations to prevent people who do not have certification from providing health services, even in the virtual environment, without proper accreditation and certificate of expertise [8]. In our study, most of the participants stated that they did not have sufficient knowledge about using telerehabilitation methods and needed education to use telerehabilitation methods. This situation may be related to the insufficient education about telerehabilitation applications for physiotherapists and this indicates the need for education and policy investments to make the telerehabilitation approach applicable in all contexts [16]. 
Our data showed that in addition to the fact that the vast majority of the participants made an effort to improve remote monitoring and telerehabilitation, some of the individuals who adopted these methods held sessions with less frequency than face-to-face interviews. Few of these individuals received payment from the patients, while those who received payment demanded a lower amount than before. These results show that these methods should be enriched, differentiated, and more importantly, they should be used within a legal framework, with the same frequency as face-to-face sessions and charged as rehabilitation services.

In Portugal, it was reported that only emergency care should be provided throughout the outbreak with regulatory restriction rules, and that discretion was left to physiotherapists in determining which cases would benefit most from their intervention [8]. It is predicted that after the inevitable cessation of treatments or decrease in intensity, a large group of patients in need of rehabilitation will have to be dealt with, the results will be evaluated in the upcoming period and the effect on overall patient function will be profound $[4,5]$. Neurorehabilitation units will need to adapt their facilities and resources, and quickly use new tools that include telerehabilitation methods in order to better manage both patients with COVID sequelae and their usual patients after the pandemic [17]. Participants in our study stated that the patient groups whose continuity of treatment decreased in the last year were pediatric patients at the highest rate, adult neurological patients and patients with musculoskeletal injuries. During this period, rehabilitation of chronic conditions such as cerebral palsy or neuromuscular pathologies was suspended in other countries, and warnings were given about the risk of reduction in future functional outcomes [6] and increased risk of care burden with mental health impairment [18]. There were proposals to urgently rethink the current and future management of children with disabilities [19-21]. In these countries, approaches such as telerehabilitation and home programs were activated differently in various fields by providing remote clinical monitoring and psychological support if necessary [22, 23]. Recent studies show that home-based programs are effective in improving the motor functions of children with cerebral palsy, which is the main cause of childhood disability worldwide [24]. Although sudden and unplanned, this process can provide an opportunity for the design of home programs and the implementation of family-centered therapies and encourage therapists to interact with the family in the context of priorities and the needs of the child's role in the home [25].

Besides the cardiovascular system being the first affected system in SARS-CoV-2, significant prevalence of neurological complications was reported $[15,26,27]$. Neurological sequelae are accompanied by clinical signs such as respiratory/pulmonary capacity loss, general weakness and postural problems, and secondary pain [15, 28, 29]. In our study, physiotherapists performed rehabilitation interventions for patients with COVID-19, and a high rate of these patients required rehabilitation due to clinical conditions such as respiratory weakness, general muscle weakness, and lower rates required rehabilitation due to fatigue, atrophy, neuropathy and post-COVID stroke. There are few clinical studies and scientific guidelines for physiotherapy and rehabilitation of patients with COVID-19 [30]. In this process, rehabilitation specialists feel as if they are navigating unknown waters; many ordinary and scientific sources of information such as reviews, guidelines or recommendations can be useful, but may be insufficient to confront the current scenario [31]. In our study, during the pandemic, a small number of physiotherapists reported that they were involved in development teams for audio-visual material for use as exercise, protocols and/or guidelines that included rehabilitation management and treatment for patients with COVID-19, and they accessed the available materials and reported that they benefited from the materials. Many of these materials are outdated within a few days or weeks, or must be changed according to specific local needs or unforeseen circumstances [31]. A reasonable option to assist rehabilitation professionals is to provide timely and continuous reports from the field, with contributions at different stages of the pandemic and in different settings or types of services; this can lead to sharing experiences, avoiding wrong practices, and spreading good practice examples [31]. The numbers of these patients and their rehabilitation content can be presented as case series in articles in the future [5].

\section{Limitations}

This study has certain limitations. One of these limitations was that no distinction was made between physiotherapists working in public and private clinics. There may be differences in the practice in each sector, such as the earnings of businesses and access 
to protective measures. Especially those who have their own workplaces (private clinics) or those working in private clinics may face situations such as taking a break from their work to protect their health or continuing to work to provide for their homes and families. In addition, the fact that the results cannot be generalized to physiotherapists in other countries is another limitation of the study. Another limitation is that, as a cross-sectional study, data were collected in a certain time period and the sudden effects of this viral pandemic may have changed the status of responders at that time; the COVID-19 outbreak continues to spread in our country and the number of cases continues to increase. Finally, the gender differences of the participants were not considered in the study, this variable can be considered as an interfering factor. Given these limitations, more research is needed in this area which includes a larger group and a variety of health professionals and their mental condition.

\section{Conclusion}

The patient groups whose continuity of treatment was reduced were mostly pediatric and adult neurological patients, and the burden that these patient groups may place on health services after restrictions are lifted should be considered. In order to alleviate the burden on clinical centers during and after the pandemic, increasing the number of resources and sessions, utilizing technological rehabilitation services for more intensive treatment, establishing new clinics and increasing the employment of physiotherapists appear to be methods that can be applied. The development and dissemination of telerehabilitation services will enable physiotherapists to reach patients with video conferencing methods and to increase the therapy intensity of patients. Therapeutic strategies can thus be adapted to minimize physical contact while providing therapeutic benefit; these digital strategies may become more common, but care must be taken to maintain the quality of the intervention provided. For this purpose, it is important to strengthen the academic and educational content about evaluation and treatment applications with the telerehabilitation method offered to rehabilitation professionals. Policy investments in pricing and mainstreaming may be needed to make the telerehabilitation approach applicable in all contexts.

Considering the needs of patients with COVID-19, it is obvious that physiotherapists need to increase their knowledge, especially within the scope of post-intensive care evaluation and treatment. Sharing experiences through ongoing reports can lead to avoidance of wrong practices and the dissemination of good practice examples; the number of patients and their rehabilitation content may be presented as case series in articles in the future. With the necessity for all these applications, there is a need to initiate research projects about the evaluation of the specific and general impact of the current pandemic in the field of physiotherapy and rehabilitation in the future.

\section{Conflict of interest}

No conflict of interest was declared by the authors.

\section{References}

[1] Boldrini P, Bernetti A, Fiore P, SIMFER Executive Committee, SIMFER Committee for International Affairs. Impact of COVID-19 outbreak on rehabilitation services and Physical and Rehabilitation Medicine physicians' activities in Italy. An official document of the Italian PRM Society (SIMFER). Eur J Phys Rehabil Med. 2020;56(3):316-318.

[2] Grasselli G, Pesenti A, Cecconi M. Critical Care Utilization for the COVID-19 Outbreak in Lombardy, Italy: Early Experience and Forecast During an Emergency Response. JAMA. 2020;323(16):1545-1546.

[3] Boldrini P, Kiekens C, Bargellesi S, et al. First impact of COVID-19 on services and their preparation. "Instant paper from the field" on rehabilitation answers to the COVID-19 emergency. Eur J Phys Rehabil Med. 2020;56(3):319- 322.

[4] Chaler J, Gil Fraguas L, Gómez García A, et al. Impact of Coronavirus disease 2019 outbreak on rehabilitation services and physical rehabilitation medicine and rehabilitation physicians' activities: perspectives from the Spanish experience. Eur J Phys Rehabil Med. 2020;56(3):369-371.

[5] Singh R, Burn J, Sivan M. A letter in response to the SIMFER document on the impact of COVID-19 on Italian rehabilitation services and activities. Eur J Phys Rehabil Med. 2020;56(3):368-369.

[6] Negrini S, Grabljevec K, Boldrini P, et al. Up to 2.2 million people experiencing disability suffer collateral damage each day of COVID-19 lockdown in Europe. Eur J Phys Rehabil Med. 2020;56(3):361-365.

[7] Minghelli B, Soares A, Guerreiro A, et al. Physiotherapy services in the face of a pandemic. Rev Assoc Med Bras (1992). 2020;66(4):491-497.

[8] Alpalhão V, Alpalhão M. Impact of COVID-19 on Physical Therapist Practice in Portugal. Phys Ther. 2020; 100(7):1052-1053.

[9] Republic of Turkey Ministry of Health. COVID-19 Information Platform. [homepage on the Internet]. c2021 [updated 2021 Jan 9; cited 2021 Mar 13]. Available from: https://covid19.saglik.gov.tr/TR-66300/covid-19-ne dir-.html

[10] Omidi L, Moradi G, Mostofi Sarkari N. Risk of COVID19 infection in workplace settings and the use of personal protective equipment. Work. 2020;66(2):377-378. 
[11] Paraskevopoulos E, Papandreou M. Systematic infection control in Greek physiotherapy practices during the COVID-19 pandemic. Work. 2020;66(2):367-370.

[12] World Confederation for Physical Therapy. Information and resources about COVID-19. [homepage on the Internet]. c2021 [updated $2021 \mathrm{Jan} 9$; cited $2021 \mathrm{Mar}$ 13]. Available from: https://www.wcpt.org/news/NovelCoronavirus-2019-nCoV

[13] Chang MC, Park D. How Should Rehabilitative Departments of Hospitals Prepare for Coronavirus Disease 2019? Am J Phys Med Rehabil. 2020;99(6):475-476.

[14] Rossato MDS, Gregorio D, de Almeida-Pedrin RR, et al. Evaluation of Dental Practices Changes During the COVID19 Pandemic. Eval Health Prof. 2021;163278721994902.

[15] Faccioli S, Lombardi F, Bellini P, Costi S, Sassi S, Pesci MC. How Did Italian Adolescents with Disability and Parents Deal with the COVID-19 Emergency? Int J Environ Res Public Health. 2021;18(4):1687.

[16] Provenzi L, Grumi S, Gardani A, et al. Italian parents welcomed a telehealth family-centred rehabilitation programme for children with disability during COVID-19 lockdown. Acta Paediatr. 2021;110(1):194-196.

[17] Juárez-Belaúnde A, Colomer Font C, Laxe S, Ríos-Lago M, Ferri Campos J. The future of neurorehabilitation after the SARS-CoV-2 pandemic. Futuro de la neurorrehabilitación tras la pandemia por el SARS-CoV-2. Neurologia. 2020;35(6):410-411.

[18] Fegert JM, Vitiello B, Plener PL, Clemens V. Challenges and burden of the Coronavirus 2019 (COVID-19) pandemic for child and adolescent mental health: a narrative review to highlight clinical and research needs in the acute phase and the long return to normality. Child Adolesc Psychiatry Ment Health. 2020;14:20.

[19] Trabacca A, Russo L. COVID-19 and child disabilities: whom to protect and how. Eur J Phys Rehabil Med. 2020;56(3):372-373.

[20] Schiariti V. The human rights of children with disabilities during health emergencies: the challenge of COVID-19. Dev Med Child Neurol. 2020;62(6):661.

[21] Jesus TS, Landry MD, Jacobs K. A 'new normal' following COVID-19 and the economic crisis: Using systems thinking to identify challenges and opportunities in disability, telework, and rehabilitation. Work. 2020;67(1):37-46.

[22] Negrini S, Kiekens C, Bernetti A, et al. Telemedicine from research to practice during the pandemic. "Instant paper from the field" on rehabilitation answers to the COVID-19 emergency. Eur J Phys Rehabil Med. 2020;56(3):327-330.

[23] Starace F, Ferrara M. COVID-19 disease emergency operational instructions for Mental Health Departments issued by the Italian Society of Epidemiological Psychiatry. Epidemiol Psychiatr Sci. 2020;29:e116.

[24] Novak I, Morgan C, Fahey M, et al. State of the Evidence Traffic Lights 2019: Systematic Review of Interventions for Preventing and Treating Children with Cerebral Palsy. Curr Neurol Neurosci Rep. 2020;20(2):3.

[25] Longo E, de Campos AC, Schiariti V. COVID-19 Pandemic: Is This a Good Time for Implementation of Home Programs for Children's Rehabilitation in Low- and Middle-Income Countries? Phys Occup Ther Pediatr. 2020;40(4):361-364.

[26] Mao L, Jin H, Wang M, et al. Neurologic Manifestations of Hospitalized Patients With Coronavirus Disease 2019 in Wuhan, China. JAMA Neurol. 2020;77(6):683-690.

[27] Bridwell R, Long B, Gottlieb M. Neurologic complications of COVID-19. Am J Emerg Med. 2020;38(7):1549.e31549.e7.

[28] Carda S, Invernizzi M, Bavikatte G, et al. The role of physical and rehabilitation medicine in the COVID-19 pandemic: The clinician's view. Ann Phys Rehabil Med. 2020;63(6):554-556.

[29] Mamo J, Feroz B, Mahmood S. Covid-19: protecting patients in hospital for neurorehabilitation and their therapists. BMJ. 2020;369:m1630.

[30] Zhong Z, Zhang J, Chen J, Liu B. Department of rehabilitation medicine in Shanghai response for coronavirus disease 2019: the impact and challenges. Eur J Phys Rehabil Med. 2020;56(3):345-346.

[31] Stam HJ, Stucki G, Bickenbach J, European Academy of Rehabilitation Medicine. Covid-19 and Post Intensive Care Syndrome: A Call for Action. J Rehabil Med. 2020;52(4):jrm00044. 